\title{
Optimal Energy Routing in Networks with Energy Cooperation
}

\author{
Berk Gurakan Omur Ozel Sennur Ulukus \\ Department of Electrical and Computer Engineering \\ University of Maryland, College Park, MD 20742 \\ gurakan@umd.edu_omur@umd.edu_ulukus@umd.edu
}

\begin{abstract}
We consider the delay minimization problem in an energy harvesting communication network with energy cooperation. We investigate the problems of optimal energy management at each node and optimal energy routing between nodes through energy cooperation. For the case without energy cooperation, each node needs to distribute its harvested energy over its outgoing links. For this problem, we show that each node should allocate more power to links with more noise power and to links through which more data flow. For the case with energy cooperation, each node needs to distribute its harvested energy among its outgoing links, and in addition, each node may transfer a portion of its harvested energy to neighboring nodes through energy cooperation. For this problem, we develop an iterative algorithm to determine the optimal energy routes in the network. We numerically observe that energy is routed from nodes with lower data loads to nodes with higher data loads.
\end{abstract}

\section{INTRODUCTION}

We consider an energy harvesting communication network with energy cooperation as shown in Fig. 1. Each node harvests energy from nature and all nodes may share a portion of their harvested energies with neighboring nodes through energy cooperation [1]. We focus on the delay minimization problem for this network. The delay on each link depends on the information carrying capacity of the link, and in particular, it decreases monotonically with the capacity of the link for a fixed data flow through it; see e.g., [2, Eqn. (5.30)]. The capacity, in turn, is a function of the power allocated to the link, and in particular, it is a monotonically increasing function of the power, for instance, through a logarithmic Shannon type capacity-power relationship [3].

We consider the capacity assignment problem for this setting [2, Section 5.4.2]. We control the energies allocated to individual outgoing links for all nodes and the energies shared by the nodes through energy cooperation [1] in order to optimize the link capacity allocations over the entire network with the goal of minimizing the overall network delay. Specifically, we consider a network with non-interfering orthogonal Gaussian links in the physical layer. We assume that the data routing topology and the data flows through the links are fixed. When energy cooperation is possible, we also assume that the energy routing topology is fixed as well. We determine the optimum energies allocated to outgoing links of the nodes and optimum amounts of energy transferred between the nodes.

This work was supported by NSF Grants CNS 13-14733, CCF 14-22111 and CCF 14-22129.

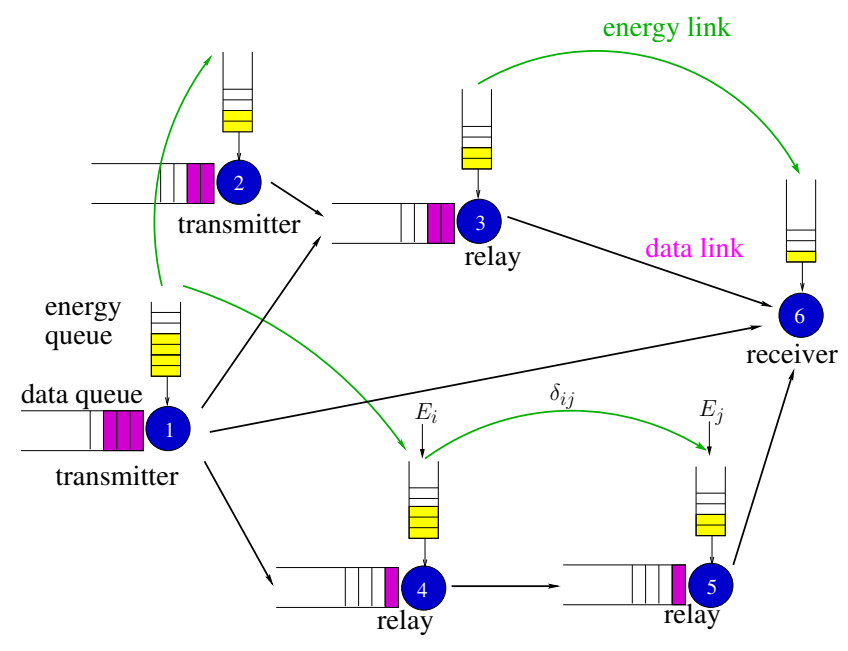

Fig. 1. System model.

We first focus on the optimal energy management problem at the nodes without energy cooperation. We show that this problem can be decomposed into individual problems, each one to be solved for a single node. For this problem, we show that more power should be allocated to links with more noise power and more data flow through them, resembling channel inversion type of power control [4]. Next, we consider the case with energy cooperation, where nodes transfer a portion of their own energies to neighboring nodes. In this case, we have the joint problem of energy routing among the network nodes and energy allocation among the outgoing links at each node. For this problem, we develop an iterative algorithm that visits all energy links sufficiently many times and decreases the network delay monotonically. We numerically observe that energy flows from nodes with lightly loaded data links to nodes with heavily loaded data links. Our work is related to and builds upon classical works on routing in communication networks [2], [5]-[9], and recent works on energy harvesting communications [10]-[12] and energy cooperation [1].

\section{Network Flow And Energy Model}

We use directed graphs to represent the network topology, and data and flow through the network. All nodes are energy harvesting, and all nodes are equipped with separate wireless energy transfer units. In this paper, for simplicity, we assume that each node harvests energy only once. 


\section{A. Network Data Topology}

We represent the data topology of the network by a directed graph which we assume is always connected. In this model, a collection of nodes, labeled $n=1, \ldots, N$, can send and receive data across communication links. A data communication link is represented as an ordered pair $(i, j)$ of distinct nodes. The presence of a link $(i, j)$ means that the network is able to send data from the start node $i$ to the end node $j$. We label the data links as $l=1, \ldots, L$. The network data topology can be represented by an $N \times L$ matrix, A, in which every entry $A_{n l}$ is associated with node $n$ and link $l$ via

$$
A_{n l}= \begin{cases}1, & \text { if } n \text { is the start node of data link } l \\ -1, & \text { if } n \text { is the end node of data link } l \\ 0, & \text { otherwise }\end{cases}
$$

We define $\mathcal{O}_{d}(n)$ as the set of outgoing data links from node $n$, and $\mathcal{I}_{d}(n)$ as the set of incoming data links to node $n$. We define $N$-dimensional vector s whose $n$th entry $s_{n}$ denotes the non-negative amount of exogenous data flow injected into the network at node $n$. On each data link $l$, we let $t_{l}$ denote the amount of flow and we call the $L$-dimensional vector $\mathbf{t}$ the flow vector. At each node $n$, the flow conservation implies:

$$
\sum_{l \in \mathcal{O}_{d}(n)} t_{l}-\sum_{l \in \mathcal{I}_{d}(n)} t_{l}=s_{n}, \quad \forall n
$$

The flow conservation law over all the network can be compactly written as:

$$
\mathbf{A t}=\mathbf{s}
$$

We define $c_{l}$ as the information carrying capacity of link $l$. Then, we require $t_{l} \leq c_{l}, \forall l$.

\section{B. Network Energy Topology}

All nodes are equipped with energy harvesting units. Each node $n$ harvests energy in the amount of $E_{n}$. We use $N$ dimensional vector $\mathbf{E}$ to denote the energy arrival vector for the system. In the energy cooperation setting, there are energy links similar to data links. An energy link is represented as an ordered pair $(i, j)$ of distinct nodes where the presence of an energy link means that it is possible to send energy from the start node to the end node. Energy links are labeled as $q=1, \ldots, Q$. Energy transfer efficiency on each energy link is denoted with $0<\alpha_{q} \leq 1$ which means that when $\delta$ amount of energy is transferred on link $q$ from node $i$ to node $j$, node $j$ receives $\alpha_{q} \delta$ amount of energy. The network energy topology can be represented by an $N \times Q$ matrix, $\mathbf{B}$, in which every entry $B_{n q}$ is associated with node $n$ and energy link $q$ via

$$
B_{n q}= \begin{cases}1, & \text { if } n \text { is the start node of energy link } q \\ -\alpha_{q}, & \text { if } n \text { is the end node of energy link } q \\ 0, & \text { otherwise }\end{cases}
$$

On each energy link $q$, we let $y_{q}$ be the amount of energy transferred. We call the $L$-dimensional vector $\mathbf{y}$ the energy flow vector. We denote by $\mathcal{O}_{e}(n)$ and $\mathcal{I}_{e}(n)$, respectively, the sets of outgoing and incoming energy links at node $n$.

\section{Communication Model and Delay Assumptions}

We assume that the delay on data link $l$ is

$$
D_{l}=\frac{t_{l}}{c_{l}-t_{l}}
$$

The delay expression in (5) is based on $\mathrm{M} / \mathrm{M} / 1$ queueing assumptions, see [2]. Each node $n$, on the transmitting edge of data link $l$, enables a capacity $c_{l}$ by expanding power $p_{l}$. These quantities are related by the Shannon formula as [3]

$$
c_{l}=\frac{1}{2} \log \left(1+\frac{p_{l}}{\sigma_{l}}\right)
$$

where all logs in this paper are with respect to base $e$. At each node $n$, the total power expanded on data and energy links are constrained by the available energy, i.e.,

$$
\sum_{l \in \mathcal{O}_{d}(n)} p_{l}+\sum_{q \in \mathcal{O}_{e}(n)} y_{q} \leq E_{n}+\sum_{q \in \mathcal{I}_{e}(n)} \alpha_{q} y_{q}, \quad \forall n
$$

Using $L$-dimensional vector $\mathbf{p}=\left(p_{1}, \ldots, p_{L}\right)$ and $\mathbf{F}=\mathbf{A}^{+}$ where $\left(A^{+}\right)_{n l}=\max \left\{A_{n l}, 0\right\}$, the energy availability constraints can be compactly written as:

$$
\mathbf{F p}+\mathbf{B y} \leq \mathbf{E}
$$

We note that we use power and energy interchangeably in (8) and in the rest of the paper by assuming slot lengths of 1 unit.

\section{iII. Capacity Assignment Problem}

We assume that the flow assignments, $t_{l}$, on all links are fixed and are serviceable by the harvested energies and energy transfers. The total delay in the network is:

$$
D=\sum_{l} \frac{t_{l}}{c_{l}-t_{l}}
$$

We formulate the capacity assignment problem, with the goal of minimizing the total delay in the network as:

$$
\begin{aligned}
\min _{c_{l}, p_{l}, y_{q}} & \sum_{l} \frac{t_{l}}{c_{l}-t_{l}} \\
\text { s.t. } & \mathbf{F p}+\mathbf{B y} \leq \mathbf{E} \\
& t_{l} \leq c_{l}, \quad \forall l
\end{aligned}
$$

By using the capacities $c_{l}$ in (6), we formulate the problem in terms of the link powers $p_{l}$ and energy transfers $y_{q}$ only as:

$$
\begin{array}{cl}
\min _{p_{l}, y_{q}} & \sum_{l} \frac{t_{l}}{\frac{1}{2} \log \left(1+\frac{p_{l}}{\sigma_{l}}\right)-t_{l}} \\
\text { s.t. } & \mathbf{F} \mathbf{p}+\mathbf{B y} \leq \mathbf{E} \\
& p_{l} \geq \sigma_{l}\left(e^{2 t_{l}}-1\right), \quad \forall l
\end{array}
$$

We solve the problem in (11) in the rest of this paper.

\section{A. Properties of the Optimal Solution}

First, we note that the objective function can be written in the form $\sum_{i} f_{i}\left(g\left(x_{i}\right)\right)$ where $f_{i}\left(x_{i}\right)=\frac{t_{i}}{x_{i}-t_{i}}$ and $g\left(x_{i}\right)=\frac{1}{2} \log \left(1+x_{i}\right)$. Since $f$ is convex and non-increasing and $g$ is concave, the resulting composition function is convex 
[13]. The constraint set is linear. Therefore, (11) is a convex optimization problem. The Lagrangian function is:

$$
\begin{aligned}
\mathcal{L}= & \sum_{l} \frac{t_{l}}{\frac{1}{2} \log \left(1+\frac{p_{l}}{\sigma_{l}}\right)-t_{l}}+\sum_{n} \lambda_{n}\left[\sum_{l \in \mathcal{O}_{d}(n)} p_{l}\right. \\
& \left.+\sum_{q \in \mathcal{O}_{e}(n)} y_{q}-E_{n}-\sum_{q \in \mathcal{I}_{e}(n)} \alpha_{q} y_{q}\right] \\
& -\sum_{l} \beta_{l}\left[p_{l}-\sigma_{l}\left(e^{2 t_{l}}-1\right)\right]-\sum_{q} \theta_{q} y_{q}
\end{aligned}
$$

The KKT optimality conditions are:

$$
\begin{aligned}
h_{l}^{\prime}\left(p_{l}\right)+\lambda_{n(l)}-\beta_{l} & =0, \quad \forall l \\
\lambda_{m(q)}-\alpha_{q} \lambda_{k(q)}-\theta_{q} & =0, \quad \forall q
\end{aligned}
$$

where $h_{l}\left(p_{l}\right) \triangleq t_{l}\left(\frac{1}{2} \log \left(1+\frac{p_{l}}{\sigma_{l}}\right)-t_{l}\right)^{-1}, n(l)$ is the beginning node of data link $l, m(q)$ and $k(q)$ are the beginning and end nodes of energy link $q$ respectively. The additional complementary slackness conditions are:

$\lambda_{n}\left(\sum_{l \in \mathcal{O}_{d}(n)} p_{l}+\sum_{q \in \mathcal{O}_{e}(n)} y_{q}-E_{n}-\sum_{q \in \mathcal{I}_{e}(n)} \alpha_{q} y_{q}\right)=0, \forall n$

$\beta_{l}\left[p_{l}-\sigma_{l}\left(e^{2 t_{l}}-1\right)\right]=0, \quad \forall l$

$\theta_{q} y_{q}=0, \quad \forall q$

We now identify some properties of the optimal power allocation in the following three lemmas.

Lemma 1 If the problem in (11) is feasible, then $\beta_{l}=0, \forall l$.

Proof: If the problem in (11) is feasible, its objective function must be bounded. Equality in the second set of constraints in (11) for any $l$ implies that the objective function is unbounded. Therefore, we must have strict inequality in those constraints for all $l$, and from (16) we conclude that $\beta_{l}=0, \forall l$.

Lemma 2 At every node $n$, the optimal power allocation amongst different outgoing data links satisfies

$$
h_{l}^{\prime}\left(p_{l}\right)=h_{m}^{\prime}\left(p_{m}\right), \quad \forall l, m \in \mathcal{O}_{d}(n)
$$

Proof: From (13) and Lemma 1 we have,

$$
h_{l}^{\prime}\left(p_{l}\right)=-\lambda_{n(l)}, \quad \forall l
$$

For outgoing data links $l, m$ that belong to the same node $n$,

$$
h_{l}^{\prime}\left(p_{l}\right)=-\lambda_{n}=h_{m}^{\prime}\left(p_{m}\right)
$$

which gives the desired result.

Lemma 3 If some energy is transferred through energy link $q$ across nodes $(i, j)$, then,

$$
h_{l}^{\prime}\left(p_{l}\right)=\alpha_{q} h_{m}^{\prime}\left(p_{m}\right), \quad \forall l \in \mathcal{O}_{d}(i), \forall m \in \mathcal{O}_{d}(j)
$$

Proof: If some energy is transferred through energy link $q$, then $y_{q}>0$, and from (17), $\theta_{q}=0$. From (14), we have,

$$
\lambda_{i}=\alpha_{q} \lambda_{j}
$$

Writing (13) for nodes $i$ and $j$, we have

$$
\begin{aligned}
h_{l}^{\prime}\left(p_{l}\right) & =-\lambda_{i}, \quad \forall l \in \mathcal{O}_{d}(i) \\
h_{m}^{\prime}\left(p_{m}\right) & =-\lambda_{j}, \quad \forall m \in \mathcal{O}_{d}(j)
\end{aligned}
$$

and the result follows from combining (22), (23) and (24).

In the following two sub-sections, we separately solve the problem for the cases of no energy transfer and with energy transfer.

\section{B. Solution for the Case of No Energy Transfer}

In the case of no energy transfer, we have $y_{q}=0, \forall q$, and the problem becomes only in terms of $p_{l}$ as stated below:

$$
\begin{array}{ll}
\min _{p_{l}} & \sum_{l} \frac{t_{l}}{\frac{1}{2} \log \left(1+\frac{p_{l}}{\sigma_{l}}\right)-t_{l}} \\
\text { s.t. } & \sum_{l \in \mathcal{O}_{d}(n)} p_{l} \leq E_{n}, \quad \forall n \\
& p_{l} \geq \sigma_{l}\left(e^{2 t_{l}}-1\right), \quad \forall l
\end{array}
$$

This problem can be decomposed into $N$ sub-problems as:

$$
\begin{array}{ll}
\min _{p_{l} \geq 0} & \sum_{n} \sum_{l \in \mathcal{O}_{d}(n)} \frac{t_{l}}{\frac{1}{2} \log \left(1+\frac{p_{l}}{\sigma_{l}}\right)-t_{l}} \\
\text { s.t. } & \sum_{l \in \mathcal{O}_{d}(n)} p_{l} \leq E_{n}, \quad \forall n \\
& p_{l} \geq \sigma_{l}\left(e^{2 t_{l}}-1\right), \quad \forall l
\end{array}
$$

Since the constraint set depends only on the powers of node $n$, there is no interaction between the nodes. Every node will independently solve the following optimization problem:

$$
\begin{array}{ll}
\min _{p_{l} \geq 0} & \sum_{l \in \mathcal{O}_{d}(n)} \frac{t_{l}}{\frac{1}{2} \log \left(1+\frac{p_{l}}{\sigma_{l}}\right)-t_{l}} \\
\text { s.t. } & \sum_{l \in \mathcal{O}_{d}(n)} p_{l} \leq E_{n} \\
& p_{l} \geq \sigma_{l}\left(e^{2 t_{l}}-1\right), \quad \forall l \in \mathcal{O}_{d}(n)
\end{array}
$$

The feasibility of (27) requires $E_{n} \geq \sum_{l \in \mathcal{O}_{d}(n)} \sigma_{l}\left(e^{2 t_{l}}-1\right)$ which we assume holds. Similar to (11), (27) is a convex optimization problem with the KKT optimality conditions:

$$
h_{l}^{\prime}\left(p_{l}\right)+\lambda=0, \quad \forall l \in \mathcal{O}_{d}(n)
$$

with the complementary slackness condition:

$$
\lambda\left(\sum_{l \in \mathcal{O}_{d}(n)} p_{l}-E_{n}\right)=0
$$

The Lagrange multipliers for the second set of constraints in (27) are not included, because similar to Lemma 1, they will 
always be satisfied with strict inequality. From (28), we have

$$
\begin{aligned}
-\lambda & =h_{l}^{\prime}\left(p_{l}\right) \\
& =\frac{-t_{l}}{2 \sigma_{l}}\left[\frac{1}{2} \log \left(1+\frac{p_{l}}{\sigma_{l}}\right)-t_{l}\right]^{-2}\left(1+\frac{p_{l}}{\sigma_{l}}\right)^{-1}
\end{aligned}
$$

After some algebraic manipulations, we have:

$$
p_{l}(\lambda)=\sigma_{l}\left(e^{2\left(W(\Delta)+t_{l}\right)}-1\right)
$$

where $\Delta=\sqrt{\frac{t_{l} e^{-2 t_{l}}}{2 \lambda \sigma_{l}}}$ and $W(\cdot)$ is the Lambert $\mathrm{W}$ function defined as the inverse of the function $w \rightarrow w e^{w}$ [14].

Next, we prove some monotonicity properties for the optimal solution, as a function of the qualities the channels and the amounts of data flows through the channels.

Lemma 4 For fixed $t_{l}, p_{l}$ is monotone increasing in $\sigma_{l}$.

Proof: By differentiating (32) and using the following [14]

$$
\frac{d W(z)}{d z}=\frac{W(z)}{z(1+W(z))}
$$

it can be verified that

$$
\frac{\partial p_{l}}{\partial \sigma_{l}}=e^{2 t_{l}} \frac{e^{2 W(\Delta)}}{1+W(\Delta)}-1>0
$$

where the last inequality follows from $e^{2 t_{l}}>1, \forall t_{l}>0$, and $\frac{e^{2 W(\Delta)}}{1+\Delta}>1, \forall \Delta>0$, proving the lemma.

This lemma shows that, for fixed data flows, more power should be allocated to channels with more noise power, similar to channel inversion power control [4].

Lemma 5 For fixed $\sigma_{l}, p_{l}$ is monotone increasing in $t_{l}$.

Proof: By differentiating (32), it can be verified that

$$
\frac{\partial p_{l}}{\partial t_{l}}=\frac{\sigma_{l}\left(W(\Delta)+2 t_{l}\right) e^{2\left(W(\Delta)+t_{l}\right)}}{t_{l}(1+W(\Delta))}>0
$$

proving the lemma.

This lemma shows that, for fixed channel qualities, more power should be allocated to links with more data flow.

Finally, we solve (27) in the following way: From the total energy constraint, we have $\sum_{l} p_{l}\left(\lambda^{*}\right)=E_{n}$. We perform a one dimensional search on $\lambda$ to find $\lambda^{*}$ that satisfies $\sum_{l} p_{l}\left(\lambda^{*}\right)=$ $E_{n}$. Once $\lambda^{*}$ is found, the optimal power allocations are found by plugging $\lambda^{*}$ in (32).

\section{Solution for the Case with Energy Transfer}

In this section, we solve for the case with energy transfer, i.e., $y_{q} \geq 0$ for some $q$. We assume that some energy $y_{q}>0$ is transferred from node $i$ to node $j$ on energy link $q$. Writing (32) for the outgoing data links of node $i$ and node $j$ we have,

$$
\begin{array}{cl}
p_{l}\left(\lambda_{i}\right)=\sigma_{l}\left(e^{2\left(W\left(\Delta_{i}\right)+t_{l}\right)}-1\right), & \forall l \in \mathcal{O}_{d}(i) \\
p_{l}\left(\lambda_{j}\right)=\sigma_{l}\left(e^{2\left(W\left(\Delta_{j}\right)+t_{l}\right)}-1\right), & \forall l \in \mathcal{O}_{d}(j)
\end{array}
$$

where $\Delta_{i}=\sqrt{\frac{t_{l} e^{-2 t_{l}}}{2 \lambda_{i} \sigma_{l}}}$ and $\Delta_{j}=\sqrt{\frac{t_{l} e^{-2 t_{l}}}{2 \lambda_{j} \sigma_{l}}}$. From (22) we have $\lambda_{i}=\alpha_{q} \lambda_{j}$. The energy causality constraints on node $i$ and $j$ are

$$
\begin{aligned}
& \sum_{l \in \mathcal{O}_{d}(i)} p_{l}\left(\lambda_{i}^{*}\right)=E_{i}-y_{q} \\
& \sum_{l \in \mathcal{O}_{d}(j)} p_{l}\left(\lambda_{j}^{*}\right)=E_{j}+\alpha_{q} y_{q}
\end{aligned}
$$

Equations (22), (38) and (39) imply

$$
\alpha_{q} \sum_{l \in \mathcal{O}_{d}(i)} p_{l}\left(\alpha_{q} \lambda_{j}^{*}\right)+\sum_{l \in \mathcal{O}_{d}(j)} p_{l}\left(\lambda_{j}^{*}\right)=\alpha_{q} E_{i}+E_{j}
$$

which can be solved by a one-dimensional search on $\lambda_{j}^{*}$.

We solve (11) by iteratively allowing energy to flow through a single link at a time provided all links are visited infinitely often. Since we do not know which energy links will be active in the optimal solution, we may need to call back any transferred energy in the previous iterations. To perform this, we keep track of transferred energy in each energy link by means of meters as in [1]. In particular, we open only one energy link $q$ at a time and whenever energy flows through link $q$, (40) must be satisfied. At every iteration, we search for $\lambda_{j}^{*}$ that satisfies (40). If no solution to (40) can be found, then previously transferred energy must be called back to the extent possible according to the meter readings. Our algorithm will converge to a local minimum because each iteration strictly decreases the objective function and bounded real monotone sequences always converge. This local minimum is the global minimum due to the convexity of the problem.

\section{Numerical Results}

In this section, we give simple numerical results to illustrate the resulting optimal policies. We study two network topologies shown in Figs. 2 and 3. For all examples, we assume $\sigma_{l}=0.1$ units $\forall l$. The slot length is of 1 unit, so that we use power and energy; rate and data interchangeably.

\section{A. Network Topology 1}

We first consider the network topology in Fig. 2 with one source, one destination and three relays in between. The data and energy links are shown and labeled as in Fig. 2 where $l_{i}$ s represent data links and $y_{q} \mathrm{~s}$ represent energy links. The fixed data flows are $\mathbf{t}=\left[t_{1}, \ldots, t_{7}\right]=$ $[2,1,0.5,0.125,2.125,0.375,0.5]$ units. The energy arrival vector is $\mathbf{E}=\left[E_{1}, \ldots, E_{4}\right]=[15,8,5,1]$ units and energy transfer efficiencies are $\boldsymbol{\alpha}=\left[\alpha_{1}, \alpha_{2}, \alpha_{3}\right]=[0.6,0.5,0.5]$.

The optimal energy transfer vector is found as $\mathbf{y}=$ $\left[y_{1}, y_{2}, y_{3}\right]=[1.82,4.75,2.85]$ units and power allocation vector after energy transfer is $\mathbf{p}=\left[p_{1}, \ldots, p_{7}\right]=$ $[11.01,2.15,0.67,0.14,9.42,0.37,0.67]$ units. Lemmas 2 and 3 can be verified numerically: $h_{l}^{\prime}\left(p_{l}\right)$ equalizes for different outgoing links of the same node, for example, on links $l_{1}$ and $l_{2}$ (Lemma 2); and where some energy is transferred, $h_{l}^{\prime}\left(p_{l}\right)$ is proportional to the energy transfer efficiency of that energy transfer link, for example, $h_{3}^{\prime}\left(p_{3}\right) / h_{1}^{\prime}\left(p_{1}\right)=\alpha_{1}$ (Lemma 3). It is interesting to note that node 4 has transferred more energy 


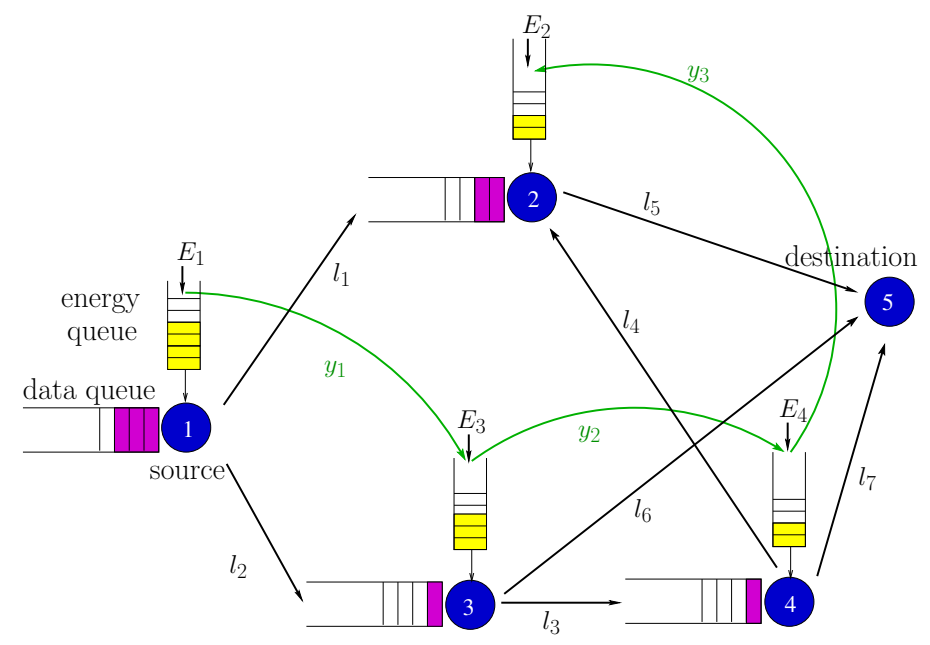

Fig. 2. Network topology 1.

than it initially had, which means that most of the transferred energy has been routed from other nodes. This is due to the high data flow on link $l_{5}$ which leads to a higher energy demand at node 2 .

\section{B. Network Topology 2}

We next consider the star topology in Fig. 3 where 5 sources are communicating with one destination similar to a multiple access scenario. The data flows are $\mathbf{t}=[0.5,2,0.5,0.5,2]$ units. The energy arrivals to all the nodes are assumed to be the same, i.e., $E_{n}=15$ units, $\forall n$. The wireless energy transfer efficiencies are $\alpha_{q}=0.5, \forall q$.

The optimal energy transfer vector is found as $\mathbf{y}=$ $[11.92,0,9.66,16.29,0]$ units and the power vector after energy transfer is $\mathbf{p}=[3.07,20.96,5.33,3.53,23.15]$ units. This system is symmetric in terms of energy arrivals, channel noises and energy transfer efficiencies, and furthermore $t_{1}=t_{3}=t_{4}$ and $t_{2}=t_{5}$. In this scenario, one might expect $p_{1}=p_{3}=p_{4}$ and $p_{2}=p_{5}$. However, in the optimal solution $p_{5}>p_{2}$. We explain the reason for this asymmetry as follows. Due to the high data loads on links $l_{2}$ and $l_{5}$, there is no incentive for these nodes to share their energy. Then, in the optimal solution, $y_{2}=y_{5}=0$ and nodes 2 and 5 act as energy sink nodes where energy is collected and not sent out. We see that node 5 has two nodes transferring energy to it while node 2 has only one node transferring energy. Then, $p_{5}>p_{2}$.

\section{CONCLUSION}

We considered the energy management and energy routing problems for delay minimization in energy harvesting networks with energy cooperation. In this network, there are data links where data flows and energy links where energy flows. For the energy management problem at each node, we showed that the allocated power increases with the noise power and the data flow amount on the outgoing link. For the energy routing problem among nodes, we developed an iterative algorithm that determines the optimal capacity allocations and energy routes in the network. In this work, we considered a single

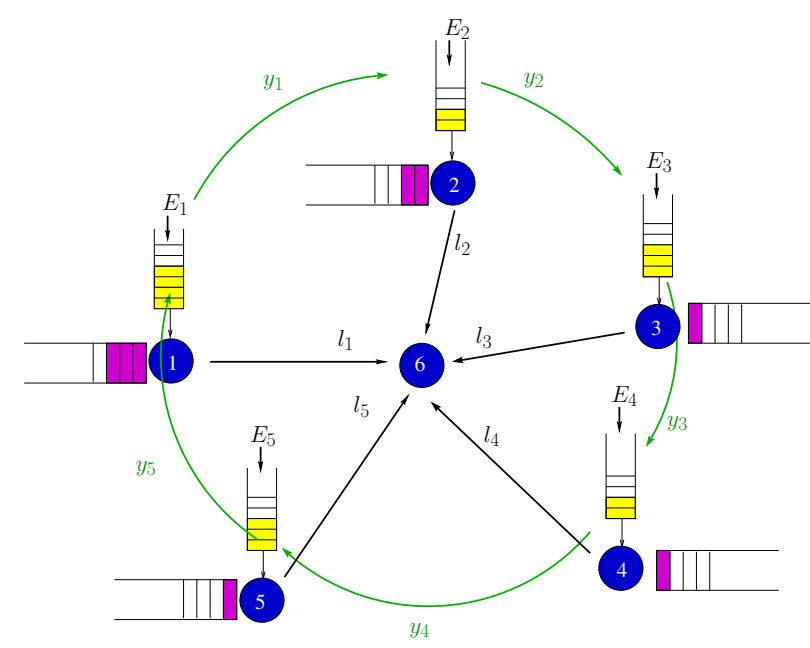

Fig. 3. Network topology 2.

energy arrival at each node. Our work can be extended for the case of multiple energy arrivals by modifying the network graph in an appropriate manner and using the developed iterative algorithm. Joint determination of data and energy routes [15] in networks with energy cooperation can be considered in future work.

\section{REFERENCES}

[1] B. Gurakan, O. Ozel, J. Yang, and S. Ulukus, "Energy cooperation in energy harvesting communications," IEEE Trans. Comm., vol. 61, pp. 4884-4898, December 2013.

[2] D. Bertsekas and R. Gallager, Data Networks. Prentice-Hall, Inc., 1992.

[3] T. M. Cover and J. A. Thomas, Elements of Information Theory. WileyInterscience, second ed., 2006.

[4] A. J. Goldsmith and P. P. Varaiya, "Capacity of fading channels with channel side information," IEEE Trans. on Inform. Theory, vol. 43, no. 6, pp. 1986-1992, 1997.

[5] D. Bertsekas, Network Optimization: Continuous and Discrete Models. Athena Scientific, 1998.

[6] B. Gavish and I. Neuman, "A system for routing and capacity assignment in computer communication networks," IEEE Trans. Comm., vol. 37, no. 4, pp. 360-366, 1989.

[7] H. H. Yen and F. S. Lin, "Near-optimal delay constrained routing in virtual circuit networks," in IEEE INFOCOM, April 2001.

[8] L. Xiao, M. Johansson, and S. Boyd, "Simultaneous routing and resource allocation via dual decomposition," IEEE Trans. Comm., vol. 52, no. 7, pp. 1136-1144, 2004.

[9] R. L. Cruz and A. V. Santhanam, "Optimal routing, link scheduling and power control in multihop wireless networks," in IEEE INFOCOM, March 2003.

[10] J. Yang and S. Ulukus, "Optimal packet scheduling in an energy harvesting communication system," IEEE Trans. Comm., vol. 60, pp. 220-230, January 2012.

[11] K. Tutuncuoglu and A. Yener, "Optimum transmission policies for battery limited energy harvesting nodes," IEEE Trans. Wireless Comm., vol. 11, pp. 1180-1189, March 2012.

[12] O. Ozel, K. Tutuncuoglu, J. Yang, S. Ulukus, and A. Yener, "Transmission with energy harvesting nodes in fading wireless channels: Optimal policies," IEEE JSAC, vol. 29, pp. 1732-1743, September 2011.

[13] S. Boyd and L. Vandenberghe, Convex Optimization. Cambridge University Press, 2004.

[14] R. M. Corless, G. H. Gonnet, D. E. Hare, D. J. Jeffrey, and D. E. Knuth, "On the lambertw function," Adv. in Comp. Math., vol. 5, pp. 329-359, December 1996.

[15] S. Cui, R. Madan, A. Goldsmith, and S. Lall, "Joint routing, MAC, and link layer optimization in sensor networks with energy constraints," in IEEE ICC, May 2005. 